\title{
以荷叶制备多级孔碳硫复合正极材料及性能研究
}

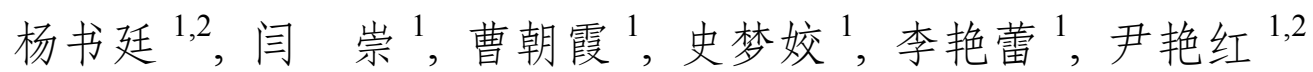

(1. 河南师范大学 化学化工学院, 新乡 453007; 2. 动力电源及关键材料国家地方联合工程实验室, 新乡 453000)

摘 要: 以荷叶为原料, 采用多阶炭化的方法, 得到高比表面积 $\left(572.1 \mathrm{~m}^{2} / \mathrm{g}\right)$ 和存在大量多级孔尤其微孔(平均孔径 $3.31 \mathrm{~nm})$ 结构居多的炭骨架，继而用高能球磨法及熔融法与单质硫进行复合制备出不同含硫量 $(48 \mathrm{wt} \%, 62 \mathrm{wt} \%$, $71 \mathrm{wt} \%$ )碳/硫复合材料。通过 XRD、FESEM、EDS 和 TG 对材料结构和形貌进行表征，结果表明硫被均匀固定在多 孔碳材料的类石墨烯层状结构和类微米棒结构中。充放电测试表明, $62 \mathrm{wt} \%$ 含硫量的复合正极材料性能表现最佳， 在 $0.1 C, 1.2 \sim 2.8 \mathrm{~V}$ 范围内充放电，首次放电比容量达 $1246 \mathrm{mAh} / \mathrm{g}, 100$ 次循环后依旧保持在 $600 \mathrm{mAh} / \mathrm{g}$, 制备出的 复合正极材料对多硫化物的 “穿梭效应” 起到了抑制作用。

关 键 词: 锂硫电池; 多阶炭化; 多级孔; 荷叶

中图分类号: TM912 文献标识码: A

\section{Preparation of Hierarchical Porous Carbon/Sulfur Composite Based on Lotus-leaves and Its Property for Li-S Batteries}

\author{
YANG Shu-Ting ${ }^{1,2}$, YAN Chong ${ }^{1}$, CAO Zhao-Xia ${ }^{1,2}$, SHI Meng-Jiao ${ }^{1}$, LI Yan-Lei ${ }^{1}$, YIN Yan-Hong ${ }^{1,2}$ \\ (1. College of Chemistry and Chemical Engineering, Henan Normal University, Xinxiang 453007, China ; 2. National \& Local \\ Engineering Laboratorg for Motive Power and Key Materials, Xinxiang 453000, China)
}

\begin{abstract}
The carbon skeleton with high specific surface $\left(572.1 \mathrm{~m}^{2} / \mathrm{g}\right)$ and large number of hierarchical pores especially micro-pores (average pore size at $3.2 \mathrm{~nm}$ ) was obtained from common lotus-leaves by using multistage carbonization method. Ball milling and melting method were used to synthesize different $\mathrm{S}$ contents (48wt\%/ $62 \mathrm{wt} \% / 71 \mathrm{wt} \%$ ) carbon/sulfur composite materials. XRD, FESEM, EDS, and TG were employed to analyze structure and morphology of the samples. The result indicated that element sulfur was uniformly accommodated in the skeletons which were composed by graphene-like layered structure and micrometer sticks. Charge/discharge tests at current density of $0.1 C$ in the voltage range of 1.2-2.8 $\mathrm{V}$ showed that the initial discharge specific capacity of the carbon/sulfur composite $(62 \mathrm{wt} \%)$ reached $1246 \mathrm{mAh} / \mathrm{g}$, and its capacity still remained $600 \mathrm{mAh} / \mathrm{g}$ even after 100 charge/discharge cycles. The results prove that the obtain composites can suppress the "shuttle effect" of polysulfide species effectively.
\end{abstract}

Key words: lithium sulfur battery; multistage carbonization; hierarchical porous pores; lotus-leaves

随着电子设备和新能源汽车的普及, 对储能领 域提出了更高的要求, 人们不断探索高性能储能电
池 ${ }^{[1-2]}$ 。锂-硫电池具有很高的理论比容量 $(1675 \mathrm{mAh} / \mathrm{g})$, 安全的工作电压 $(1.5 \sim 2.5 \mathrm{~V})$, 以及硫元素在地壳中

收稿日期: 2015-08-11; 收到修改稿日期：2015-09-19

基金项目: 河南省重点科技攻关项目(122102210234); 河南省教育厅高校科技创新人才支持计划(14HASTIT004); 河南省 教育厅高等学校重点科研项目(15A150012)

Key Scientific and Technological Project of Henan Province (122102210234); Program for Science \& Technology Innovation Talents in Universities of Henan Province (14HASTIT004); Foundation of He'nan Educational Committee (15A 150012$)$

作者简介: 杨书廷(1962-)，男，博士，教授.E-mail: shutingyang@foxmail.com 
的丰度较大，且近乎无毒的优点而有望成为新型电 池 $^{[3]}$ 。但是由于硫的电子绝缘性 $\left(25^{\circ} \mathrm{C}\right.$ 条件下导电率 仅为 $5 \times 10^{-30} \mathrm{~S} / \mathrm{cm}$ )、充放电过程中多硫化物在电解 液中的溶解和伴随的 “穿梭效应”及硫的体积膨胀 $(80 \%)$, 会导致电池容量的快速衰退和电池首圈充 放电库伦效率低 $(70 \% \sim 80 \%)^{[3-4]}$ 。目前, 通过优化正极 合成微孔或介孔 ${ }^{[3-9]} \mathrm{C} / \mathrm{S}$ 复合材料,包覆导电材料 ${ }^{[10-12]}$ 构建导电网络框架, 改进隔膜和电解液 ${ }^{[13-15]}$, 对锂 负极采取包覆、粉化 ${ }^{[16-17]}$ 等方法, 抑制锂枝晶的生长 是提高锂硫电池循环稳定性的常用途径。

探索具有多孔结构和高比面积的正极材料与硫 复合来提高电池的循环稳定性, 是一种简单有效的 方法 ${ }^{[3-9]}$ 。Zhang 等 ${ }^{[5]}$ 用甘蔗作为碳源, 通过升华硫 和碳球混合热处理将硫嵌入碳球中, 制备出平均孔 径极为狭小的微孔碳一硫复合物, 复合材料表现出 优异的循环性能, 在 $400 \mathrm{~mA} / \mathrm{g}$ 的电流密度下充放电 500 次循环后仍然能保持 $650 \mathrm{mAh} / \mathrm{g}$ 。Zhang 等 ${ }^{[7]}$ 以 荔枝为原料，通过炭化和刻蚀制备出硫含量 $60 \mathrm{wt} \%$ 的碳/硫复合材料, 在 $200 \mathrm{~mA} / \mathrm{g}$ 电流密度下 100 次 循环后容量保持在 $700 \mathrm{mAh} / \mathrm{g}$ 左右。Zhao 等 ${ }^{[8]}$ 受到 鱼鳞的启发, 以其为碳源制备出孔径分布在 2 5 nm 左右的碳材料, 当硫含量为 $66.7 \mathrm{wt} \%$ 时, 电流密度 在 $1675 \mathrm{~mA} / \mathrm{g}$ 条件下经过 70 圈充放电循环后, 容量 仍为 $600 \mathrm{mAh} / \mathrm{g}$ 。

本工作选用廉价易得的生物质材料荷叶作为碳 源, 在气氛炉氮气氛中进行不同温度梯度的炦烧, 制备出具有分级孔结构并保存了生物质炭骨架的材 料, 采用球磨法和熔融法制备碳硫复合材料, 并研 究了该复合材料的结构特征及其在锂硫电池中的性 能表现。

\section{1 实验方法}

\section{1 材料的制备}

多级孔碳材料的制备: 将采集的荷叶表面用蒸 馏水洗净, 放入 $80^{\circ} \mathrm{C}$ 的鼓风干燥箱中干燥 $48 \mathrm{~h}$, 干 燥样品研磨成粉在气氛炉(南京大学产)氮气氛中进 行梯度升温, 四个阶段升温区间分别为 $25^{\circ} \mathrm{C} \sim 290^{\circ} \mathrm{C} \sim$ $450^{\circ} \mathrm{C} \sim 580^{\circ} \mathrm{C} \sim 850^{\circ} \mathrm{C}$, 升温速率为 $5^{\circ} \mathrm{C} / \mathrm{min}$, 在 $290^{\circ} \mathrm{C}$ 、 $450^{\circ} \mathrm{C} 、 580^{\circ} \mathrm{C}$ 分别保温 $2 \mathrm{~h}$ 。待自然冷却至室温后, 用 $1 \mathrm{~mol} / \mathrm{L}$ 的稀盐酸 $(\mathrm{A} \mathrm{R})$ 浸泡样品 $6 \mathrm{~h}$ 后, 去离子水 洗涤至中性并干燥, 即得到多级孔碳材料。

碳/硫复合物 $(\mathrm{C} / \mathrm{S})$ 的制备: 升华硫(A.R.上海产) 和碳材料分别按质量比为 $2: 2 、 2: 1 、 3: 1$ 的比例 混合后球磨 $2 \mathrm{~h}$ (转速为 $600 \mathrm{r} / \mathrm{min}$ ); 将球磨产物放置
于瓷舟中，在管式炉中氮气保护气氛下以 $3^{\circ} \mathrm{C} / \mathrm{min}$ 升 温至 $155^{\circ} \mathrm{C}$, 保温 $6 \mathrm{~h}$; 再以 $3^{\circ} \mathrm{C} / \mathrm{min}$ 升温至 $300^{\circ} \mathrm{C}$, 保 温 $3 \mathrm{~h}$, 自然冷却至室温后得到碳/硫 $(\mathrm{C} / \mathrm{S})$ 复合材料。

\section{2 结构和形貌表征}

用 D8 Advance 型 X 射线衍射仪(德国 Bruker 公 司产)对多孔碳材料和碳/硫复合材料进行物相分析; 使用 SU-8010 型场发射扫描电子显微镜(FESEM) (日 本产)进行表面形貌结构的观察和 EDS 分析。

用 STA-449-F3 同步热分析仪(上海产)测定碳/ 硫复合物中 $\mathrm{S}$ 的含量; 用 Tristar- II 比表面积和孔隙 率分析仪(美国产)测试氮气等温吸脱附曲线, $P / P_{0}$ 为 $0.005 \sim 1.0$ 。

\section{3 电池的组装}

按照碳/硫复合物 : SP : PVDF=7 : 2: 1 的质 量比混料球磨 $2 \mathrm{~h}$, 以 $\mathrm{N}$-甲基吡咯烷酮(NMP)(上海 产)为溶剂在玛瑙研钵中进行调浆, 将浆料均匀涂 覆在 $20 \mu \mathrm{m}$ 厚的铝箔(上海产, 99.9\%)上, $60^{\circ} \mathrm{C}$ 真空 (真空度为 $0.09 \mathrm{MPa}$ ) 条件下干燥 $24 \mathrm{~h}$ 后冲成 $\phi 12 \mathrm{~mm}$ 的圆形正极片, 活性物质的载量大约为 $1.1 \mathrm{mg} / \mathrm{cm}^{2}$ 。

以金属锂片为负极，制备的复合材料为正极, $1 \mathrm{~mol} / \mathrm{L} \mathrm{LiTFSI} / \mathrm{DME}: \mathrm{DOL}=1: 1$ 溶液为电解液, $0.1 \mathrm{~mol} / \mathrm{L} \mathrm{LiNO}_{3}$ 为添加剂, 在布劳恩手套箱(德国产) 中组装成 CR2032 型扣式电池, 静置 $12 \mathrm{~h}$ 后进行充 放电测试。

\section{4 充放电性能测试}

采用 CT2001A 型充放电测试仪(武汉金诺)进行 充放电性能测试, 充放电倍率为 $0.1 C$ (电流密度为 $167 \mathrm{~mA} / \mathrm{g}$ ), 电压为 $1.2 \sim 2.8 \mathrm{~V}$ 。采用 CHI760B 电化 学工作站(上海辰华)进行循环伏安和交流阻抗测试, 扫描电压范围为 $1.2 \sim 2.8 \mathrm{~V}$ (扫速为 $0.05 \mathrm{mV} / \mathrm{s}$ )。

\section{2 结果与讨论}

\section{1 结构与形貌}

\subsubsection{XRD 表征}

图 1 示出了升华硫、多级孔碳材料及碳/硫复合 材料的 XRD 图谱。从图 1 曲线 a 可以看到升华硫具 有多个不同强度的特征衍射峰, 对比标准图谱 (JCPDS 08-0247), 升华硫以强晶态形式存在。从图 1 曲线 $\mathrm{b}$ 可知, 多极孔碳材料在 $2 \theta=25^{\circ}$ 附近有明显 宽的鼓包肩峰, 在 $2 \theta=43^{\circ}$ 有轻微的小鼓包峰, 这是 碳材料的特征衍射峰, 代表典型的无定形类石墨晶 体的(002)平面和(100)平面。从图 1 曲线 $\mathrm{c}$ 可知, 碳/ 硫复合材料在 $2 \theta=20^{\circ} \sim 30^{\circ}$ 出现无定形碳的类石墨晶 型衍射峰, 硫的特征衍射峰与标准图谱吻合得比较 
好, 表明此复合材料中硫通过热处理的方法已固定 在多级孔的炭骨架材料中, 由于多级孔炭骨架是开 放式结构, 并不能将硫完全容纳在孔腔之内, 部分 硫吸附在外层孔道中, 因此仍能探测到硫部分晶面 的衍射峰。

\subsection{2 不同硫含量下的碳/硫复合材料的表征}

图 2 是三种不同含硫量 $(48 \mathrm{wt} \% 、 62 \mathrm{wt} \% 、 71 \mathrm{wt} \%)$ 的碳/硫复合材料热重曲线图。从三条热重曲线上看, 在 $350^{\circ} \mathrm{C}$ 时硫元素仍没有完全损失, 表明碳材料与硫 有较强的键合作用 ${ }^{[16-17]}$, 这在一定程度上抑制了多 硫化物的穿梭。

\subsection{3 比表面积及孔径分布表征}

图 3(a)和图 3(b) 是含硫量为 $62 \mathrm{wt} \%$ 的碳/硫复合 物的氮气等温吸脱附曲线, 表 1 给出了两种材料的 孔结构参数。如图 3(a)所示, 多孔碳材料低压区曲 线偏向 $\mathrm{Y}$ 轴, 说明有微孔存在; 而中压区有明显滞 后环，表明存在介孔结构; 高压区有轻微上扬,

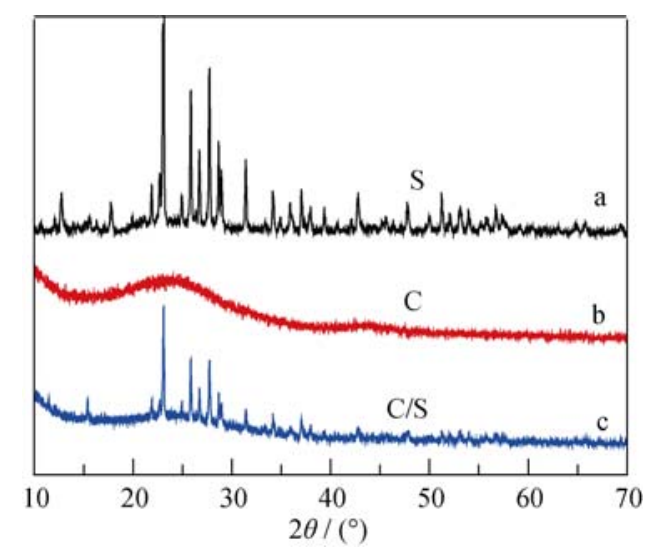

图 1 样品的 XRD 图谱

Fig. 1 XRD patterns of samples

(a) Sulfur; (b) Hierarchical porous carbon; (c) $\mathrm{C} / \mathrm{S}$ composites

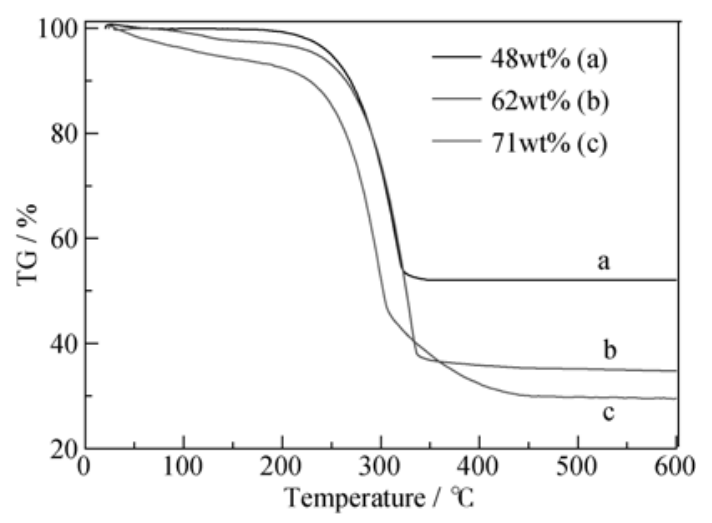

图 2 不同硫含量的复合材料热重曲线图

Fig. 2 TGA profiles of $\mathrm{C} / \mathrm{S}$ composite with different sulfur contents

(a) $48 \mathrm{wt} \% \mathrm{~S}$; (b) $62 \mathrm{wt} \% \mathrm{~S}$; (c) $71 \mathrm{wt} \% \mathrm{~S}$
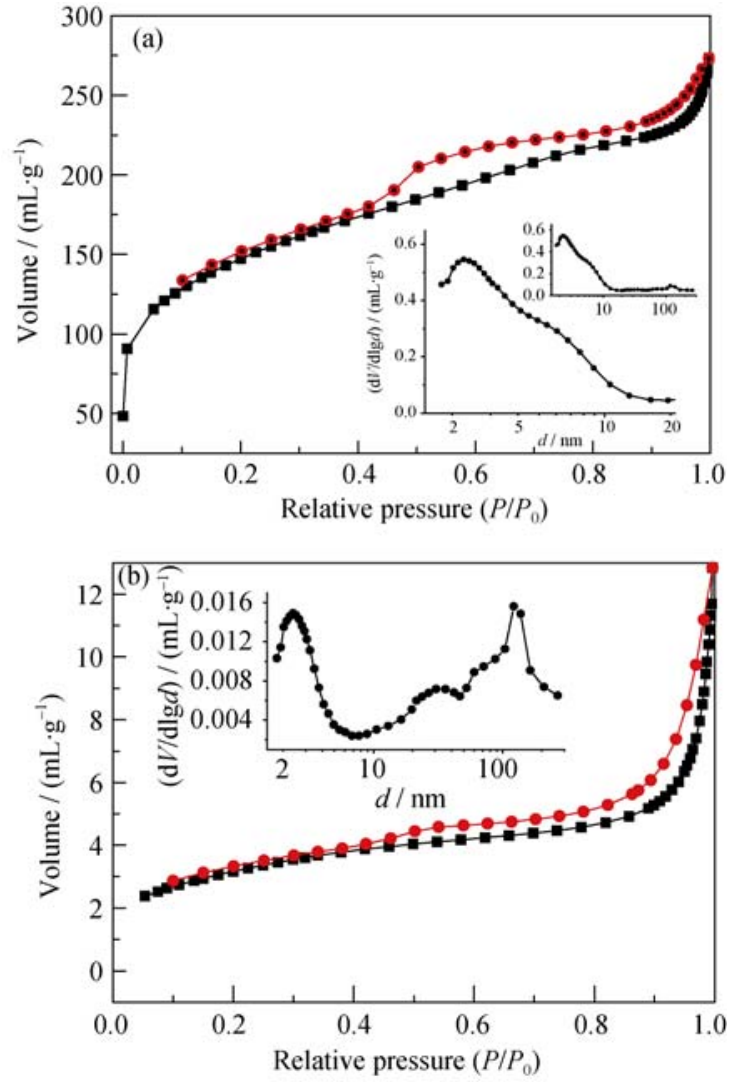

图 3 多级孔碳材料(a)和碳/硫复合材料(b)的吸脱附等温曲线 及孔径分布

Fig. 3 Adsorption-desorption isothermal and inset pore size distribution curves of carbon (a) and carbon/sulfur composite materials (b)

表 1 多孔碳材料和碳/硫复合材料的孔结构参数

Table 1 Pore parameters derived from $\mathrm{N}_{2}$ adsorption data

\begin{tabular}{cccc}
\hline Samples & $\begin{array}{c}\text { Surface } \\
\text { area } /\left(\mathrm{m}^{2} \cdot \mathrm{g}^{-1}\right)\end{array}$ & $\begin{array}{c}\text { Pore } \\
\text { diameter } / \mathrm{nm}\end{array}$ & $\begin{array}{c}\text { Pore } \\
\text { volume } /\left(\mathrm{cm}^{3} \cdot \mathrm{g}^{-1}\right)\end{array}$ \\
\hline $\mathrm{C}$ & 572.1 & 3.31 & 0.52 \\
$\mathrm{C} / \mathrm{S}$ & 11.5 & 0.04 & 0.08 \\
\hline
\end{tabular}

表明存在大孔或者裂隙孔 ${ }^{[14]}$ 。图 3(b)为碳/硫复合物 的氮气吸脱附曲线, 其比表面仅为 $11.5 \mathrm{~m}^{2} / \mathrm{g}$, 表明 硫在熔融条件下扩散并固定在炭骨架的多级孔隙中, 填充了材料的多级孔容, 图 3(b)内嵌图也表明复合 材料以介孔为主。

\subsubsection{FESEM 表征}

图 4 是酸处理前后的多极孔碳材料的 FESEM 照片。从图 4(a)低倍率 FESEM 照片显示, 材料中存 在两种形貌: 一种是类似石墨烯片层且厚度为微米 级的层状结构(图 4(c)更易观测到), 另一种是表面 附着大量白色颗粒狼牙棒形貌的结构。EDS 分析棒 状结构上的白色颗粒含 $\mathrm{Ca} 、 \mathrm{Na} 、 \mathrm{C} 、 \mathrm{O}$ 等生物体内 必需元素, 可能来自 $\mathrm{CaCO}_{3}$ 和 $\mathrm{Na}_{2} \mathrm{CO}_{3}$, 稀盐酸处理 后白色颗粒消失(图 4(b))。经过 ICP 分析测定, 酸处 

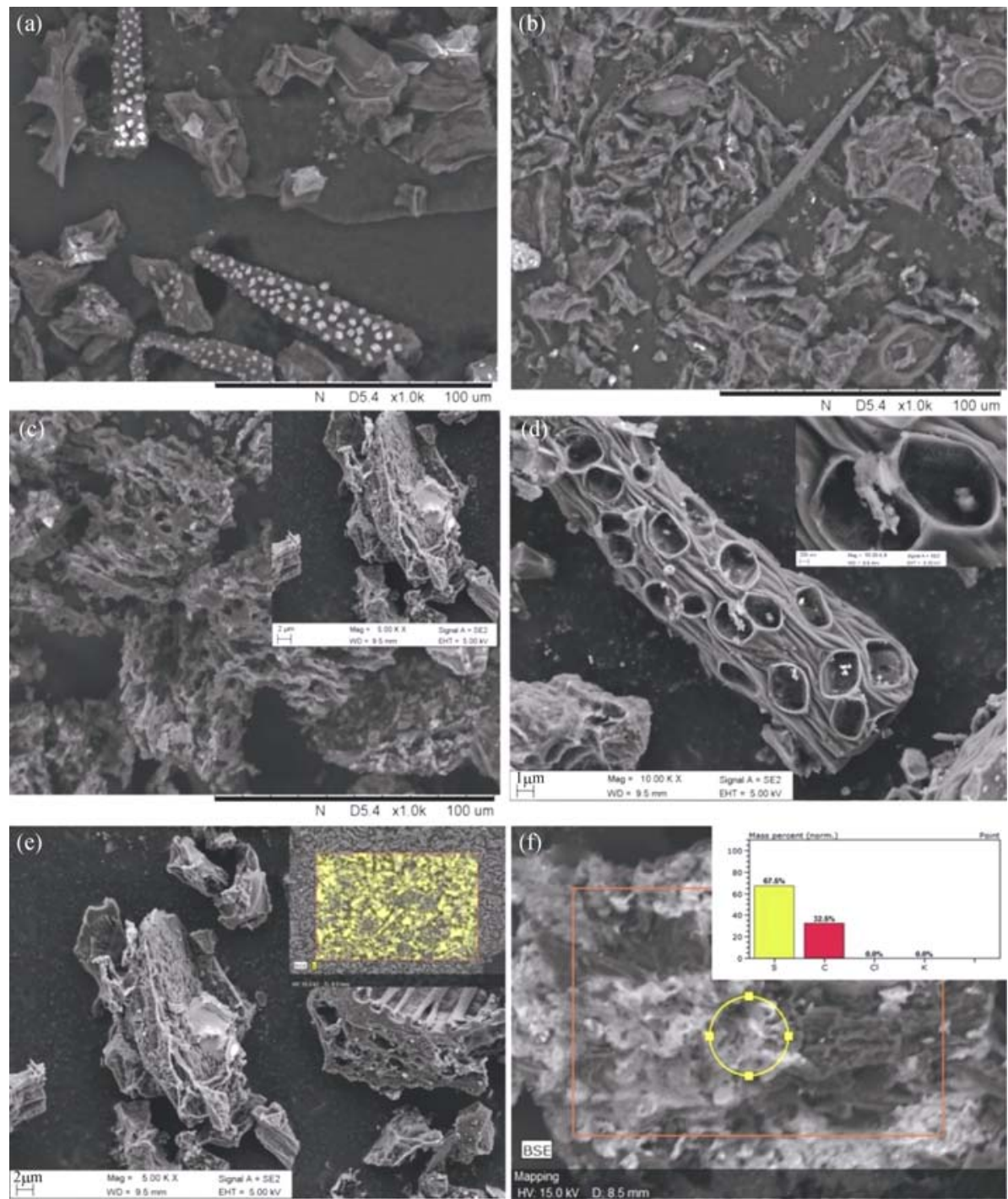

图 4 多级孔碳材料的 FESEM 照片 $(\mathrm{a}, \mathrm{b}, \mathrm{c}, \mathrm{d})$ 及能谱分析图 $(\mathrm{e}, \mathrm{f})$

Fig. 4 FESEM images of hierarchical porous carbon $(\mathrm{a} \sim \mathrm{d})$ and EDS analysis $(\mathrm{e}, \mathrm{f})$

(a) Morphology before acid treatment; (b) Morphology after acid treatment; (c) Similar graphene layered structure;

(d) Similar micrometer sticks; (e) Qualitative analysis of EDS; (f) Quantitative analysis of EDS

理前多级孔碳材料含有 $3.23 \mathrm{wt} \% \mathrm{Ca}$ 和 $2.96 \mathrm{wt} \% \mathrm{Na}$; 稀盐酸处理后含有 $0.037 \mathrm{wt} \% \mathrm{Ca}$ 和 $0.025 \mathrm{wt} \% \mathrm{Na}$ 。高 倍率 FESEM 照片(图 4(c)和图 4(d)) 显示出碳材料良 好在孔结构, EDS 定量分析(图 4(f))随机选取的截面 的含硫量达到 $67.55 \mathrm{wt} \%$ (表 2)。

\section{2 电性能测试}

\subsection{1 不同硫含量的碳/硫复合材料电性能测试}

图 5 是三种不同硫含量 $(48 \mathrm{wt} \%$ 、62 wt \% 、 $71 \mathrm{wt} \%)$

表 2 碳/硫复合材料的 EDS 定量分析表

Table 2 EDS quantitative analysis of $\mathrm{C} / \mathrm{S}$ composite

\begin{tabular}{ccc}
\hline Element & wt $\%$ & at $\%$ \\
\hline Sulfur16 K-series & 67.55 & 43.81 \\
Carbon6 K-series & 32.45 & 56.19 \\
Chlorine17 K-series & 0.00 & 0.00 \\
\hline
\end{tabular}

的正极复合材料电化学充放电循环性能曲线, 可以看出 硫含量为 $62 \mathrm{wt} \%$ 的正极材料表现最佳, $0.1 C(167 \mathrm{~mA} / \mathrm{g})$ 倍率条件下, 电池的首次放电比容量达到 $1246 \mathrm{mAh} / \mathrm{g}$, 100 圈充放电循环后仍稳定在 $600 \mathrm{mAh} / \mathrm{g}$, 电性能表现 与类似碳材料相当 ${ }^{[16]}$ 。

电池的放电比容量前 3 圈充放电衰退较快，这 是由于在电池循环过程中, 负极材料中的 $\mathrm{Li}^{+}$通过 隔膜与 $\mathrm{S}_{8}$ 或者 $\mathrm{S}_{6}{ }^{2-}$ 结合成易溶于电解液的多硫化物 $\mathrm{Li}_{2} \mathrm{~S}_{x}(4 \leqslant x \leqslant 8)$, 少量长链多硫化物会堵塞孔道或损 失到电解液中, 造成容量的不可逆损失。复合多孔 材料中并非硫含量越高越好， $71 \mathrm{wt} \%$ 含硫量的正极 材料循环稳定性表现较差, 这是由于随着硫含量的 增加, 大量孔隙被填充，充放电过程中的体积膨胀 严重抑制了其电性能表现 ${ }^{[15-17]}$ 。

从图 6 可以看到，正极材料的放电平台为 2.3 
和 $2.1 \mathrm{~V}$, 且 $2.1 \mathrm{~V}$ 放电平台曲线明显长于 $2.3 \mathrm{~V}$, 与 相关文献报道一致 ${ }^{[3]}$ 。图 7 为 $62 \mathrm{wt} \%$ 含硫量正极材 料的不同倍率电性能图, $5 C$ 充放电倍率条件下, 放 电比容量依然维持在 $470 \mathrm{mAh} / \mathrm{g}$ 。

\subsection{2 碳/硫复合材料的循环伏安测试}

图 8 是含硫量为 $62 \mathrm{wt} \%$ 的正极复合材料的循环 伏安曲线，曲线展示的首圈、第 3 圈、第 4 圈和第 5 圈的循环伏安曲线均可以观察到一个在 $2.6 \mathrm{~V}$ 附近 较为尖锐的氧化峰和两个在 $1.6 \mathrm{~V}$ 和 $2.3 \mathrm{~V}$ 附近较为 平滑的还原峰。 $2.6 \mathrm{~V}$ 氧化峰对应 $\mathrm{Li}_{2} \mathrm{~S}$ 转变成 $\mathrm{Li}_{2} \mathrm{~S}_{8}$ 的过程; $2.3 \mathrm{~V}$ 还原峰的出现表明 $\mathrm{S}_{8}$ 首先被还原为长 链多硫化物 $\mathrm{Li}_{2} \mathrm{~S}_{x}(4 \leqslant x \leqslant 8)$, 多硫化物在 $1.6 \mathrm{~V}$ 附近 进一步被还原成硫化物 $\left(\mathrm{Li}_{2} \mathrm{~S}_{2}, \mathrm{Li}_{2} \mathrm{~S}\right)$ 。

从图 8 还可以看到, 还原峰和氧化峰都随着循 环次数的增加而发生偏移, 表明在首次充放电强烈 极化作用后，后续的电化学反应趋于稳定，第 4、5 圈循环伏安曲线基本重合, 可见炭化后的分级多孔 结构对硫和多硫化物的固定发挥了重要作用。

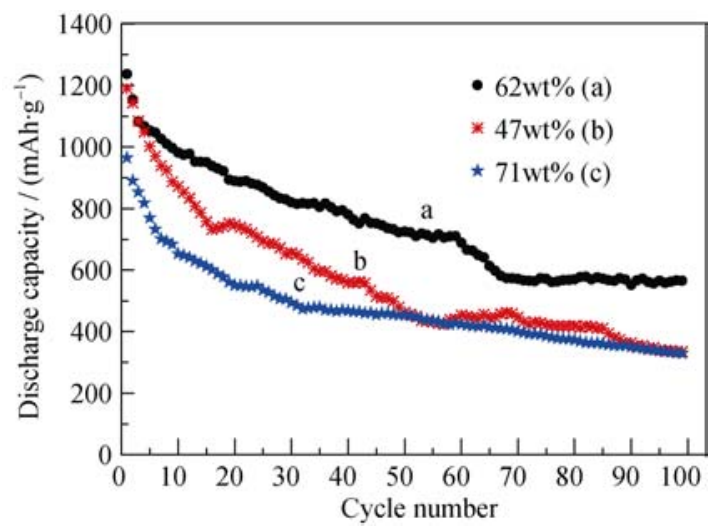

图 5 不同含硫量 $(48 \mathrm{wt} \% 、 62 \mathrm{wt} \% 、 71 \mathrm{wt} \%)$ 的碳/硫复合材料 循环充放电性能

Fig. 5 Cycling performance of $\mathrm{C} / \mathrm{S}$ composite with different contents of sulfur (a: $62 \mathrm{wt} \% \mathrm{~S} ; \mathrm{b}: 48 \mathrm{wt} \% \mathrm{~S} ; \mathrm{c}: 71 \mathrm{wt} \% \mathrm{~S}$ )

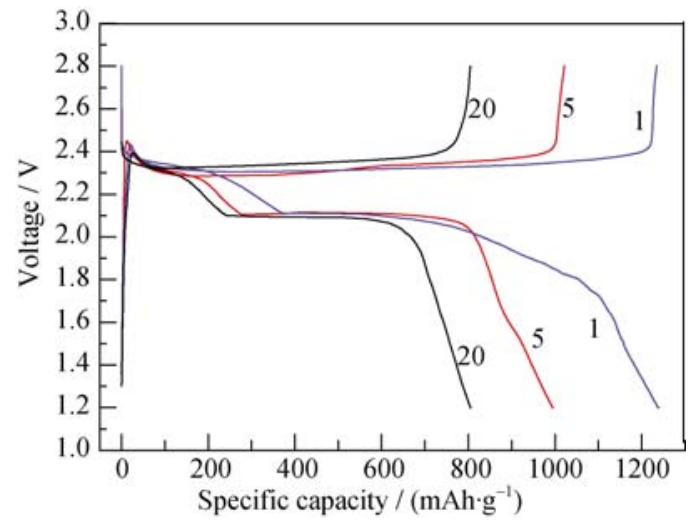

图 $662 \mathrm{wt} \%$ 含硫量复合材料的第 1 圈、第 5 圈和第 20 圈的 充放电曲线

Fig. 6 Galvanostatic charge-discharge curves of the 1st, 5th, 20th charge-discharge process for $\mathrm{C} / \mathrm{S}$ composite with $62 \mathrm{wt} \%$ sulfur

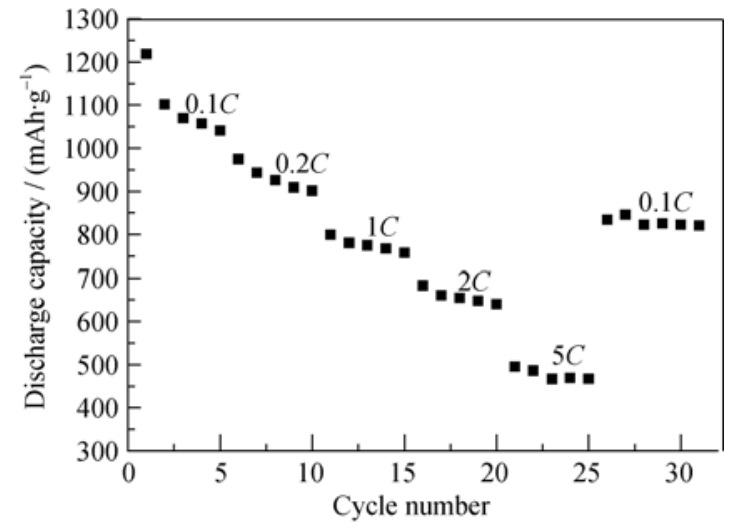

图 $762 \mathrm{wt} \%$ 含硫量复合材料的倍率性能图

Fig. 7 Rate performance of $\mathrm{C} / \mathrm{S}(62 \mathrm{wt} \%)$ composite

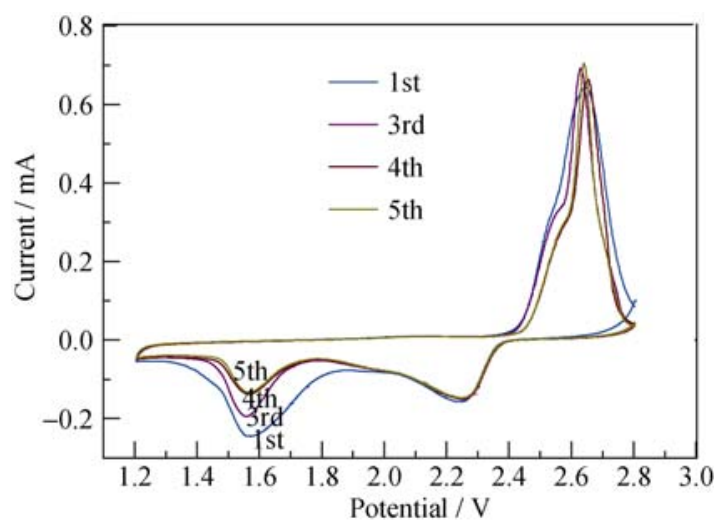

图 $862 \mathrm{wt} \%$ 含硫量复合材料在扫速为 $0.05 \mathrm{mV} / \mathrm{s}$ 的循环伏安 曲线

Fig. $8 \mathrm{CV}$ profiles of $\mathrm{C} / \mathrm{S}$ composite with $62 \mathrm{wt} \%$ sulfur at scanning rate of $0.05 \mathrm{mV} / \mathrm{s}$

\section{3 结论}

实验以原料来源广泛的荷叶为碳源，通过分阶 煅烧的方法得到具有多级孔结构的炭骨架材料，材 料的比表面积为 $572.1 \mathrm{~m}^{2} / \mathrm{g}$, 将多极孔碳材料与硫 复合制备了不同硫含量 $(48 \mathrm{wt} \% 、 62 \mathrm{wt} \% 、 71 \mathrm{wt} \%)$ 的 碳/硫复合正极材料。复合材料中保留了荷叶的骨架, 并将硫固定住，含 $62 \mathrm{wt} \%$ 硫的复合材料的电性能表 现较好, $0.1 C$ 倍率充放电测试中首次放电比容量 达 $1246 \mathrm{mAh} / \mathrm{g}, 100$ 圈循环之后仍能保持 $600 \mathrm{mAh} / \mathrm{g}$, 在 $5 C$ 倍率下, 其放电比容量依然达到 $470 \mathrm{mAh} / \mathrm{g}$, 呈现出良好的电化学性能。碳材料的加入不仅提供 了良好的导电网络框架, 其孔结构还能够容纳放电 的终极产物硫化锂, 对多硫化物的穿梭效应起到抑 制作用。

\section{参考文献:}

[1] GWON H, HONG J, KIM H, et al. Recent progress on flexible 
lithium rechargeable batteries. Energy \& Environmental Science, 2014, 7(2): 538-551.

[2] RUI XU, LU JUN, AMINEK. Progress in mechanistic understanding and characterization techniques of Li-S batteries. Advanced Energy Materials, 2015, doi:10.1002/aenm.201500408.

[3] YIN YA-XIA, XIN SEN, GUO YU-GUO, et al. Lithium-sulfur batteries: electrochemistry, materials, and prospects. Angew Chem. Int. Ed. Engl., 2013, 52(50): 13186-13200.

[4] MANTHIRAM A, FU YONG-ZHU, CHUNG SHENG-HENG, et al. Rechargeable lithium-sulfur batteries. Chemical Review, 2014, 114(23): 11751-11787.

[5] ZHANG B, QIN X, LI G.R, et al. Enhancement of long stability of sulfur cathode by encapsulating sulfur into micropores of carbon spheres. Energy \& Environmental Science, 2010, 3(10): $1531-1537$.

[6] ZHENG GUANG-YUAN, YANG YUAN, CUI YI, et al. Hollow carbon nanofiber-encapsulated sulfur cathodes for high specific capacity rechargeable lithium batteries. Nano Letters, 2011, 11(10): 4462-4467.

[7] ZHANG SONG-TAO, ZHENG MING-BO, LIN ZI-XIA, et al. Activated carbon with ultrahigh specific surface area synthesized from natural plant material for lithium-sulfur batteries. J. Mater. Chem. A, 2014, 2(38): 15889-15896.

[8] ZHAO SHENG-RONG, LI CHENG-MING, WANG WEI-KUN, et al. A novel porous nanocomposite of sulfur/carbon obtained from fish scales for lithium-sulfur batteries. J. Mater. Chem. A, 2013, 1(10): 3334-3339.

[9] YE HUAN, YIN YA-XIA, GUO YU-GUO, et al.Tuning the porous structure of carbon hosts for loading sulfur toward long lifespan cathode materials for Li-S batteries. J. Mater. Chem. A, 2013, 1(22): $6602-6608$
[10] WU YI-HAN, XU CHUN-MEI, GUO JIN-XIN, et al. Enhanced electrochemical performance by wrapping graphene on carbon nanotube/sulfur composites for rechargeable lithium-sulfur batteries. Materials Letters, 2014, 137: 277-280.

[11] LI BIN, LI SONG-MEI, LIU JIAN-HUA, et al. Vertically aligned sulfur-graphene nanowalls on substrates for ultrafast lithium-sulfur batteries. Nano Letters, 2015, 15(5): 3073-3079.

[12] ZHOU GUANG-MING, PEI SONG-FEI, CHENG HUI-MING, et al. A graphene-pure-sulfur sandwich structure for ultrafast, long-life lithium-sulfur batteries. Advanced Materials, 2014, 26(4): 625-631.

[13] HUANG JIA-QI, ZHANG QIANG, PENG HONG-JIE, et al. Ionic shield for polysulfides towards highly-stable lithium-sulfur batteries. Energy \& Environmental Science, 2014, 7(1): 347-353.

[14] HUANG JIA-QI, ZHUANG TING-ZHOU, ZHANG QIANG, et al. Permselective graphene oxide membrane for highly stable and anti-self-discharge lithium-sulfur batteries. ACS Nano, 2015, 9(3): 3002-3011.

[15] YAO HONG-BIN, YAN KAI, LI WEI-YANG, et al. Improved lithium-sulfur batteries with a conductive coating on the separator to prevent the accumulation of inactive S-related species at the cathode-separator interface. Energy \& Environmental Science, 2014, 7(10): 3381-3390.

[16] CHENG XIN-BING, PENG HONG-JIE, HUANG JIA-QI, et al. Dual-phase lithium metal anode containing a polysulfide induced solid electrolyte interphase and nanostructured graphene framework for lithium-sulfur batteries. ACS Nano, 2015, 9(6): 6373-6382.

[17] ZHENG GUANG-YUAN, LEE SEOK-WOO, LIANG ZHENG, et al. Interconnected hollow carbon nanospheres for stable lithium metal anodes. Nat Nanotechnol, 2014, 9(8): 618-623. 